\title{
A layered edge-on circumstellar disk around HK Tau B`
}

\author{
G. Duchêne ${ }^{1}$, F. Ménard ${ }^{2}$, K. Stapelfeldt ${ }^{3}$, and G. Duvert ${ }^{2}$ \\ 1 Department of Physics and Astronomy, UCLA, Los Angeles, CA 90095-1562, USA \\ 2 Laboratoire d'Astrophysique, Observatoire de Grenoble, Université Joseph Fourier, BP 53, 38041 Grenoble Cedex 9, France \\ 3 Jet Propulsion Laboratory, California Institute of Technology, 4800 Oak Grove Drive, Pasadena, CA 91109, USA
}

Received 1 July 2002 / Accepted 19 December 2002

\begin{abstract}
We present the first high angular resolution $1.4 \mathrm{~mm}$ and $2.7 \mathrm{~mm}$ continuum maps of the T Tauri binary system HK Tau obtained with the Plateau de Bure Interferometer. The contributions of both components are well disentangled at $1.4 \mathrm{~mm}$ and the star previously known to host an edge-on circumstellar disk, HK Tau B, is elongated along the disk's major axis. The optically bright primary dominates the thermal emission from the system at both wavelengths, confirming that it also has its own circumstellar disk. Its non-detection in scattered light images indicates that the two disks in this binary system are not parallel. Our data further indicate that the circumprimary disk is probably significantly smaller than the circumsecondary disk. We model the millimeter thermal emission from the circumstellar disk surrounding HK Tau B. We show that the disk mass derived from scattered light images cannot reproduce the $1.4 \mathrm{~mm}$ emission using opacities of the same population of submicron dust grains. However, grain growth alone cannot match all the observed properties of this disk. We propose that this disk contains three separate layers: two thin outer surfaces which contain dust grains that are very similar to those of the ISM, and a disk interior which is relatively massive and/or has experienced limited grain growth with the largest grains significantly smaller than $1 \mathrm{~mm}$. Such a structure could naturally result from dust settling in a protoplanetary disk.
\end{abstract}

Key words. stars: formation - stars: planetary systems: protoplanetary disks - stars: pre-main sequence stars: individual: HK Tau

\section{Introduction}

Circumstellar dusty disks are primarily studied to constrain the relevant timescales and various phases of the planetary formation process, from submicron interstellar medium (ISM) dust material to $\mathrm{km}$-sized bodies. The dramatic differences between disks surrounding 10-20 Myr-old zero-age main sequence stars such as $\beta$ Pic (Lagrange et al. 2000 and references therein) and those around the much younger T Tauri stars strongly points toward an evolutionary process that occurs within $\lesssim 10^{7} \mathrm{yrs}$. $\mathrm{T}$ Tauri stars are thus obvious targets for studying the very first stages of disk evolution and, more specifically, the growth of dust grains in protoplanetary disks from ISM-sized particules to significantly larger ones.

The dust component of circumstellar disks can be traced through its thermal continuum emission. This is especially straightforward at millimeter wavelengths, at which the disk is almost entirely optically thin. Such studies have been performed for over a decade and first hinted at the presence of

Send offprint requests to: G. Duchêne,

e-mail: duchene@astro.ucla.edu

* Based on observations carried out with the IRAM Plateau de Bure Interferometer. IRAM is supported by INSU/CNRS (France), MPG (Germany) and IGN (Spain). "large" dust grains, as evidenced by "abnormal" dust emissivity properties (Beckwith et al. 1990, hereafter B90). However, disk masses and maximum grain size are currently poorly constrained due to the uncertain grain shape and composition (Pollack et al. 1994; Henning \& Stognienko 1996). Furthermore, the low spatial resolution of most radio observations only rarely allows direct studies of the structure of the disks nor do they resolve the tight visual binary systems commonly found among pre-main sequence stars (Duchêne 1999 and references therein). It is also possible to study the dust grain population of circumstellar disks through high contrast, high-angular resolution imaging of scattered light in the environment of pre-main sequence stars. Such observations, however, are only sensitive to those grains whose sizes are within a factor of a few of the wavelength. The presence (or absence) of much smaller/bigger grains is not constrained by scattered light images. Only the combination of the thermal and scattering approaches can fully determine the properties of dust in circumstellar disks.

HK Tau is a 2". 4 pre-main sequence binary system located in the nearby Taurus star-forming region $(D \approx 140 \mathrm{pc}$, Bertout et al. 1990). The primary star presents the usual caracteristics of a classical T Tauri star surrounded by a circumstellar accretion disk (Cohen \& Kuhi 1979; Kenyon \& Hartmann 1995). However, the secondary component, which was discovered 
by Cohen \& Kuhi (1979), is remarkably faint at visible/nearinfrared wavelengths. B90 and Beckwith et al. (1991, hereafter BS91) first detected continuum millimeter and submillimeter emission from the system and estimated a first disk mass, $M_{\mathrm{d}} \sim 10^{-2} M_{\odot}$, although their large beam sizes did not resolve the binary.

The HK Tau system was later observed at high angular resolution in the near-infrared by Koresko (1998, hereafter K98) and by Stapelfeldt et al. (1998, hereafter S98) through deep HST/WFPC2 visible imaging. HK Tau A appeared as a bright point source while HK Tau $B^{1}$ offered a dramatically different shape, that of an extended nebulosity, about $210 \mathrm{AU}$ across, with a central dark lane. Both interpreted these images as the signature of an optically thick circumstellar disk seen almost edge-on that completely obscures the central star and only lets scattered photons reach the observer. They further estimated that the total disk mass needed to reproduce these images assuming ISM-like dust grains is 1-2 orders of magnitude smaller than that derived by B90 and BS91. More recently, D'Alessio et al. (2001, hereafter DACH01) modeled the disk of HK Tau B, trying to match simultaneously the millimeter continuum flux of B90 and the morphology of the disk as seen in optical scattered light. They assumed a disk mass several times larger than that estimated by B90 and BS96 and, allowing the grain size distribution to vary, found a reasonable match to the data for a maximum grain size between $1 \mathrm{~mm}$ and $1 \mathrm{~m}$. The large discrepancies with the conclusions of S98 and K98 raised doubts about the actual disk's structure and dust content.

However, to properly estimate the disk mass or to conclude that dust grains have grown from ISM-like particules, it is necessary to remove the ambiguity induced by the unresolved millimeter measurement of HK Tau from B90. In this paper, we present new $1.4 \mathrm{~mm}$ and $2.7 \mathrm{~mm}$ images obtained with the IRAM Plateau de Bure Interferometer with an angular resolution that allows us to resolve the binary system at the shortest wavelength. The observations and results are described in Sects. 2 and 3 respectively, while a model of the $1.4 \mathrm{~mm}$ emission is presented in Sect. 4. In Sect. 5, we evaluate the implications of our observations for the dust content of the disk surrounding HK Tau B as well as for the geometrical properties of the whole system. Finally, we summarize our findings in Sect. 6.

\section{Observations and data reduction}

The interferometric data presented in Fig. 1 were obtained with the IRAM-Plateau de Bure interferometer (Guilloteau et al. 1992). The array was used in compact configurations with 4 antennas on 8 September 1998 (configuration 4D1), and 5 antennas on both 17 December 1998 (configuration 5C2), and 5 April 1999 (configuration 5D). The baselines ranged from $24 \mathrm{~m}$ to $176 \mathrm{~m}$. The measured beam size in the final synthesized maps are $1^{\prime \prime} .5 \times 11^{\prime \prime} .4$ at position angle $15^{\circ}$ at $1.4 \mathrm{~mm}(220 \mathrm{GHz})$ and $3 . \prime 7 \times 33^{\prime \prime} 3$ at position angle $23^{\circ}$ at

\footnotetext{
${ }^{1}$ In some early studies this component was named HK Tau/c. We adopt here the convention proposed by the IAU and name the secondary component HK Tau B.
}

Table 1. Flux densities and source sizes measured in the CLEANed maps. The uncertainties quoted here for the flux densities are those associated to the fitting process and do not include the $10 \%$ flux calibration uncertainties; these are however included in the flux densities quoted in the text. The deconvolved source sizes, $a$ and $b$, are the intrinsic $F W H M$ of each source along their major and minor axes respectively. Upper limits are $3 \sigma$ for all quantities.

\begin{tabular}{cccccc}
\hline \hline $\begin{array}{c}\lambda \\
(\mathrm{mm})\end{array}$ & object & $\begin{array}{c}F_{v} \\
(\mathrm{mJy})\end{array}$ & $\begin{array}{c}a \\
\left({ }^{\prime \prime}\right)\end{array}$ & $\begin{array}{c}b \\
\left({ }^{\prime \prime}\right)\end{array}$ & $\begin{array}{c}\text { PA } \\
\left({ }^{\circ}\right)\end{array}$ \\
\hline 1.4 & $\mathrm{~A}$ & $27.7 \pm 0.6$ & $<0.5$ & $<0.5$ & - \\
& $\mathrm{B}$ & $16.2 \pm 1.2$ & $0.89 \pm 0.12$ & $<0.5$ & $46 \pm 8$ \\
2.7 & $\mathrm{~A}$ & $6.3 \pm 0.4$ & $<1$ & $<1$ & - \\
& $\mathrm{B}$ & $<1.5$ & - & - & - \\
\hline
\end{tabular}

$2.7 \mathrm{~mm}(110 \mathrm{GHz})$. Three spectral bands of $160 \mathrm{MHz}$ each at $1.4 \mathrm{~mm}$ and two such spectral bands at $2.7 \mathrm{~mm}$ were used. Two more units of the correlator were tuned to observe lines of carbon monoxyde simultaneously with the thermal continuum: ${ }^{13} \mathrm{CO}(1 \rightarrow 0)$ and ${ }^{13} \mathrm{CO}(2 \rightarrow 1)$ at $110.2 \mathrm{GHz}(10 \mathrm{MHz}$ bandwidth) and $220.4 \mathrm{GHz}(20 \mathrm{MHz})$ respectively. None of them was detected.

We used $0415+379$ and $0528+134$ as phase calibrators. The rms phase noise was $15^{\circ}$ to $40^{\circ}$ at $1.4 \mathrm{~mm}$ and $8^{\circ}$ to $20^{\circ}$ at $2.7 \mathrm{~mm}$. This phase noise introduces a position error of less than 0.1 . The flux scale was calibrated by observing the sources MWC 349, 3C 84 and 3C 454.4 and its associated uncertainty is about $10 \%$. Relative fluxes within a map can be obtained with a much better accuracy. The GILDAs software package was used to reduce the data. The continuum maps were produced using uniform weighting of the visibilities. We CLEANed the images with a Clark algorithm using a maximum of 200 loops of gain 0.1 each and setting the convergence level at $0.1 \%$ of the peak value.

\section{Results}

Table 1 summarizes all the measurements that were extracted from our datasets. The flux densities and positions of the two sources were obtained through a fit to the CLEANed images in the Fourier space. The primary, which is unresolved at both wavelengths was fit with a point source. The secondary was fit with a point source at $2.7 \mathrm{~mm}$ and with an elliptical Gaussian at $1.4 \mathrm{~mm}$. At this shorter wavelength, HK Tau B appears more elongated than the interferometer beam and a point source fit systematically results in larger residuals. From the fit to the $1.4 \mathrm{~mm}$ data, we derive a separation $\left(2{ }^{\prime \prime} 44 \pm 0 . ' 05\right)$ and position angle $\left(171^{\circ} .7 \pm 1.1\right.$, measured East of North) between the two centroids that are in excellent agreement with previous astrometric results (Monetti \& Zinnecker 1991, S98). Although the $2.7 \mathrm{~mm}$ image seems to be extended roughly along the binary orientation at the lowest contour (see Fig. 1), the map obtained after subtraction of the primary shows that the residuals at the location of the secondary are not larger than several patches of noise in the image. We therefore derived a $3 \sigma$ upper limit to the actual $2.7 \mathrm{~mm}$ flux density of HK Tau B from a fit to the 

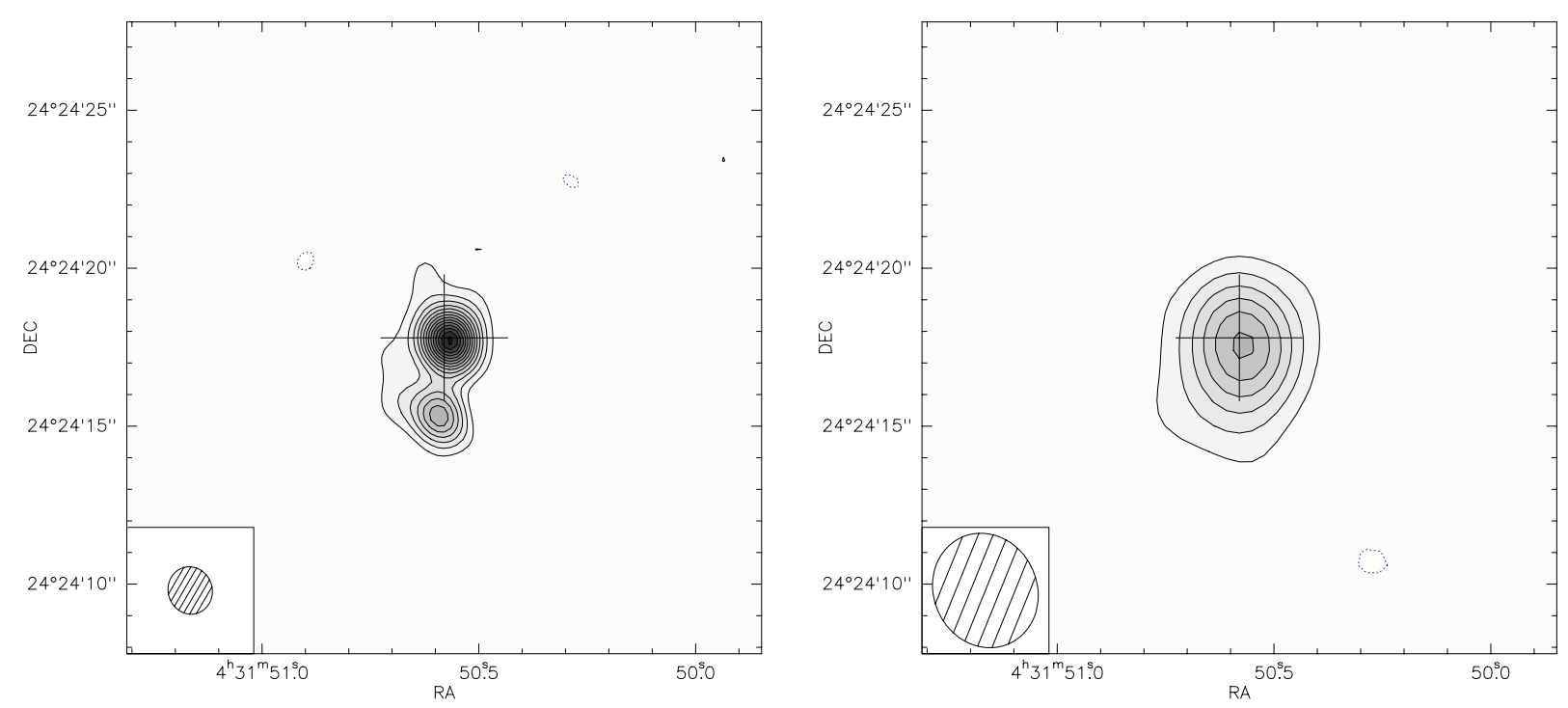

Fig. 1. Final CLEANed maps of HK Tau at $1.4 \mathrm{~mm}$ (left) and $2.7 \mathrm{~mm}$ (right). Both images are $20^{\prime \prime}$ on a side. The corresponding CLEANed beam is presented in the small inserts. The lowest contour in both images represents the $2 \sigma$ noise level $(2 \mathrm{mJy} / \mathrm{beam}$ and $1 \mathrm{mJy} / \mathrm{beam}$ at $1.4 \mathrm{~mm}$ and $3 \mathrm{~mm}$ respectively); following contours are $1,2,3, \ldots$ times this level. The cross indicates the expected location of HK Tau A from Herbig $\&$ Bell (1988), which is less than 0'. 4 away from the strongest millimeter source.

data in which we fixed the separation and position angle of the binary to the values derived from the $1.4 \mathrm{~mm}$ data.

The sum of the two components' fluxes at $1.4 \mathrm{~mm}$ is $43.9 \pm$ $4.6 \mathrm{mJy}$, in excellent agreement with B90's previous measurement of the unresolved system $(41 \pm 5 \mathrm{mJy})$. Furthermore, we find that, although HK Tau A is the dominant source in the system, HK Tau B accounts for about one third of the system's flux. From our data, we measure the spectral index ${ }^{2}$ of HK Tau A to be $\alpha_{v}(A)=2.1 \pm 0.2$. To derive a lower limit for the spectral index of HK Tau B, we conservatively adopt a uniform probability distribution for its $2.7 \mathrm{~mm}$ flux density in the range $0-1.5 \mathrm{mJy}$ and conclude that $\alpha_{v}(\mathrm{~B})>3.2$ at the $99.7 \%$ $(3 \sigma)$ confidence level.

Roughly speaking, the intrinsic size of a source adds in quadrature with the beam size to produce the observed $F W H M$ of the object. An increase of 0 ' $^{\prime} 1$ from the beam size can be detected in our data. Because HK Tau A is not resolved in our data, we infer that the size of its $1.4 \mathrm{~mm}$ emission is smaller than $70 \mathrm{AU}(F W H M)$. On the contrary, HK Tau B is extended along its major axis but unresolved along the minor axis. We derive a size of $125 \pm 17 \mathrm{AU}$, a value smaller than the $210 \mathrm{AU}$ disk diameter observed in the visible (S98). The difference is likely an effect of sensitivity limit of the interferometer to thermal dust emission. The position angle along which the elliptical Gaussian lies nicely matches the direction of the dark lane seen in scattered light images $\left(40^{\circ}, \mathrm{S} 98, \mathrm{~K} 98\right)$.

\section{Model of the millimeter emission from HK Tau B}

\subsection{Nature of the emission}

Millimeter emission from T Tauri stars is most frequently thermal in nature, and it has been modelled as such in the past

${ }^{2}$ This index, $\alpha_{v}$, is defined by the relation $F_{v} \propto v^{\alpha_{\nu}}$ between 1.4 and $2.7 \mathrm{~mm}$. (e.g., B90). However, non-thermal mechanisms, such as freefree and gyro-synchrotron emissions, can represent a non negligible fraction of the observed millimeter flux (e.g., V 773 Tau; Dutrey et al. 1996, hereafter D96). Since variable non-thermal continuum emission at $6.2 \mathrm{~cm}$ was detected from HK Tau B in the past (O'Neal et al. 1990), we discuss this possibility below.

The absence of variability in the $1.4 \mathrm{~mm}$ flux density of the unresolved system suggests that both sources are stable over several years. This tends to indicate that non-thermal emission, if present, is not dominant even though the preponderant emission from HK Tau A could strongly dilute the effects of variability in HK Tau B in the single dish measurements. Furthermore, the lower limit to the spectral index of HK Tau B between 1.4 and $2.7 \mathrm{~mm}$ is typical of optically thin thermal emission from a circumstellar disk (BS91, D96). Finally, the $1.4 \mathrm{~mm}$ emission appears to be elongated along the disk semimajor axis, whereas non-thermal emission would be confined to a very small, unresolved, volume surrounding the central star (free-free emission in the stellar chromosphere) or elongated along an axis perpendicular to the disk (synchrotron emission in a jet). We therefore conclude that most of the $1.4 \mathrm{~mm}$ we have detected from HK Tau B is the result of thermal emission from dust grains in its circumstellar disk.

\subsection{Model and results}

\subsubsection{Objectives}

To fully constrain the mass, structure and dust content of a circumstellar disk, one ultimately needs images at all wavelengths. Prior to our observations, the binary system had only been resolved shortward of $3.5 \mu \mathrm{m}$. All flux measurements in the thermal regime, i.e., at longer wavelengths, combined the flux of both stars. Building an SED for HK Tau B is not currently possible, and previous models of the thermal emission of this source suffered from this limitation. 
Our goal is to present an updated model of the disk around HK Tau B that takes into account the new data of Sect. 3. Our thermal emission model is similar in philosophy to the ones presented by B90 and D96. However, the analytical models presented in the past assumed geometrically thin disks and are invalid for edge-on disks. Although our model is relatively simple, its assumptions are adequate for a direct comparison with the scattered light model presented in S98. Therefore, differences in the disk properties inferred from these models, e.g., disk mass, temperature or dust content, will indicate the limits of our understanding of this circumstellar disk.

\subsubsection{Description of the model}

To check the relevance of the disk model derived by S98, we adopt here the same disk structure, parametrized as follows: a total gas+dust mass $M_{\mathrm{d}}$, a surface density $\Sigma(r) \propto r^{p}$ and a temperature $T(r)=T_{0}\left(r / r_{0}\right)^{q}$. The disk is assumed to be vertically isothermal and under vertical hydrostatic equilibrium. The dust and gaseous components of the disk are assumed to be perfectly mixed, i.e., they have the same temperature and density structures. Furthermore, the dust properties are assumed to be constant throughout the disk and a gas-to-dust mass ratio of 100:1 is assumed. We adopt a disk outer radius of $105 \mathrm{AU}$ and an inclination of $84^{\circ}$ (S98). The disk inner radius is set to $0.5 \mathrm{AU}$ but has a negligible influence on the result of our model, as the mass enclosed inside this radius (hence the associated thermal emission) is extremely small in all models. Finally, we initially assume a disk temperature of $T_{0}=8 \mathrm{~K}$ at $r_{0}=50 \mathrm{AU}$, which has been determined from the disk scale height estimated by $\mathrm{S} 98$.

We create synthetic $1.4 \mathrm{~mm}$ emission maps by integrating along the observer's line of sight the observed flux density from independent contiguous cells, which is determined by their volume density, temperature, opacity and optical depth to the observer. The resulting images are then convolved with an elliptical Gaussian kernel representing the interferometer primary beam to compare them to our data. One of the most crucial parameters in deriving the millimeter flux density of a disk is the choice of the dust opacity. Following B90, we adopt a total opacity of $\kappa_{v}(1.4 \mathrm{~mm})=0.02 \mathrm{~cm}^{2} / \mathrm{g}$ which takes into account the assumed gas-to-dust mass ratio. As noted by these authors, this value is an order of magnitude larger than those derived for small ( $a \leq 0.25 \mu \mathrm{m})$, compact silicate grains, but seems to be representative of the dust present in T Tauri circumstellar disks. Possible explanations for this discrepancy include a significant growth of the dust grains in circumstellar disk (B90, BS91) or a dominant presence of submicron fractal grains (Miyake \& Nakagawa 1993; Suttner \& Yorke 2001).

We explored a range of values for $M_{\mathrm{d}}, p, q$ and $T_{0}$ in an attempt to fit simultaneously the observed $1.4 \mathrm{~mm}$ flux density from HK Tau B as well as its apparent intrinsic size at this wavelength. The latter is evaluated in our model as the squareroot difference between the $F W H M$ of the disk and that of the interferometer beam. We checked that this method mimics adequately the data analysis procedures by calculating the Fourier transform of the image of several models, sampling them with the $u v$ coverage of the interferometer beam corresponding to our observational configuration and fitting the resulting datasets with the same routines as during data reduction. The disk sizes and fluxes agreed within a few percent in all cases.

\subsubsection{Model results}

The disk mass derived for HK Tau B by $\mathrm{S} 98\left(10^{-4} M_{\odot}\right)$ yields a $1.4 \mathrm{~mm}$ flux density of about $0.1 \mathrm{mJy}$, two orders of magnitude smaller than the observed one. Also, the apparent disk size produced by this model is too small compared to our observations. The best fit for both quantities is obtained for a total disk mass of $M_{\mathrm{d}} \approx 2 \times 10^{-2} M_{\odot}$ in our model. Larger masses result in $1.4 \mathrm{~mm}$ flux densities that are too large.

The disk mass needed to fit the thermal emission properties of HK Tau B is excluded by the scattered light images as it would produce a dark lane that is much too wide compared to what is observed assuming ISM-like optical opacities and well mixed grains. The apparent width of this dark lane basically measures the distance between the upper and lower $\tau_{\lambda} \approx 1$ surfaces, and is proportional to the product $\kappa_{\lambda} M_{\mathrm{d}}$. Given an adopted opacity for scattering, the total disk mass is therefore determined rather accurately in the case of edge-on disks (e.g., Burrows et al. 1996). We therefore conclude that the scattered light models of S98 and the present thermal emissiom models for HK Tau B yield solutions that are not compatible with each other. We further discuss this discrepancy in Sect. 5.1.

The disk mass derived here is similar to that derived by B90. This was expected as the loss of a factor of three in the observed $1.4 \mathrm{~mm}$ flux density has been compensated for by the lower disk temperature assumed in our model ( $8 \mathrm{~K}$ vs. $25 \mathrm{~K}$ in B90's model). Assuming a higher dust temperature of $T_{0}=32 \mathrm{~K}$ at $50 \mathrm{AU}$, we infer a total disk mass of $\approx 2 \times 10^{-3} M_{\odot}$. This value reduces the discrepancy between scattered light and thermal emission analyses but it is not fully satisfying. The gas scale height for $T_{0}=32 \mathrm{~K}$ is twice the value derived for the dust by S98. This could only be reconciled to the HST images by removing the assumption that gas and dust are fully mixed vertically, in which case the dust would have settled toward the midplane with respect to the gas. However, maintaining a thermal equilibrium between the two populations is then a difficult task, as the starlight only heats up the dust which, in turn, keeps the gas warm through collisions.

We also find that the radial surface density and/or temperature profiles in the disk must be relatively shallow to match the observed ratio between the apparent size of the disk at $1.4 \mathrm{~mm}$ and its actual size $\left(F W H M_{1.4 \mathrm{~mm}} / 2 R_{\mathrm{d}} \approx 60 \%\right)$. Quantitatively, $p+q \gtrsim-1$ is required to reproduce the apparent disk size. Models $\mathrm{C}$ and $\mathrm{D}$ from S98, which provide the best matches to the $0.8 \mu \mathrm{m}$ scattered light images, have $p+q \approx-1.1$ and $\approx-1.9$ respectively. Therefore, their model $\mathrm{D}$ seems too centrally condensed to reproduce the $1.4 \mathrm{~mm}$ extent of the disk.

\section{Discussion}

\subsection{Structure of the circumstellar disk of HK Tau B}

We investigate here the various disk models for HK Tau B that could match simultaneously the scattered light and thermal 
emission observations of this disk. The disk models used here and in S98 are similar to those usually used in studies of premain sequence circumstellar disks. In the past, they have produced satisfying models of several disks (HH 30: Burrows et al. 1996; GG Tau: Guilloteau et al. 1999, McCabe et al. 2002; IRAS 04158+2805: Ménard et al. 2002). However, they fail to reproduce the observations of $\mathrm{HK}$ Tau $\mathrm{B}$, calling for an unusual dust model or disk structure for this source.

To reconcile the $1.4 \mathrm{~mm}$ flux density of HK Tau B with the narrow width of its dark lane in visible and near-infrared images, the ratio of the optical-to-radio opacities must be modified by two orders of magnitudes from the values assumed here and in S98: either the millimeter opacity is much larger, or the optical one is much smaller. Fractal grains are unable to solve this dilemma, as they have larger opacities than compact grains at both millimeter and optical/near-infrared wavelengths (Suttner \& Yorke 2001). On the other hand, grain growth in the disk can account for such large changes in dust opacities: if the dust grain size extends continuously up to mm-sized particules, the $1.4 \mathrm{~mm}$ millimeter opacity is enhanced with respect to submicron ISM-like mixtures while the optical/near-infrared one is greatly reduced because the mass contained in $\sim 0.1-2 \mu \mathrm{m}$ grains (i.e., the most efficient scatterers) is effectively diminished for a given disk mass. A disk mass on the order of a few times $10^{-3} M_{\odot}$, i.e., intermediate between the current scattered light and thermal emission estimates, would then be favored.

There are however at least two observational constraints that appear to rule out such a strong grain growth in this disk. First, a grain size distribution extending up to $a_{\max } \sim 1 \mathrm{~mm}$ has a millimeter spectral index ${ }^{3}$ of $\alpha_{v} \sim 2.5-3.0$ (DACH01, Wood et al. 2002), which is smaller than our $3 \sigma$ lower limit for HK Tau B. The observed spectral index implies that the grain size distribution does not extend beyond $\sim 200 \mu \mathrm{m}$ at most (Fig. 3 in DACH01). Second, the analysis of scattered light images have allowed to estimate an average asymmetry parameter of the dust grains, $g_{I}=0.65$ (S98). This value is similar to those derived for other pre-main sequence disks (Burrows et al. 1996; Krist et al. 2002). Furthermore, it is very similar to the value derived by Witt et al. (1990) for a small interstellar cloud which presumably contains only submicron particules, whose absorption properties match the well-known interstellar extinction curve (Mathis \& Whiffen 1989; Kim et al. 1994; Zubko et al. 1998). However, $g$ is a strong function of grain size, especially for those grains with $a \sim \lambda$, with larger grains being more forward-throwing. If there were a significant population of such grains, uniformly distributed throughout the disk of HK Tau B, the value of $g$ inferred from scattered light images would therefore be significantly larger than what is observed elsewhere. The relatively large $2.2 \mu \mathrm{m}$ polarization rate measured by Jensen et al. (2000) for HK Tau B also points towards a dominant population of small grains in the disk since large grains $(a \gg \lambda / 2 \pi)$ result in largely unpolarized scattered light.

In summary, we conclude that the dust population traced by scattered light in the disk surrounding HK Tau B does not

\footnotetext{
3 The slightly different wavelength ranges used in various studies to determine the spectral indices may somewhat modify the spectral index estimates but changes are not expected to exceed $0.1-0.2$.
}

contain dust grains that are a few times larger $(\sim 1-3 \mu \mathrm{m})$ than those found in other pre-main sequence disks nor than in a small interstellar cloud. We acknowledge that the presence of much bigger grains without intermediate-size particles (i.e., two separate size populations) cannot be established at this stage. However, it is unclear why that intermediate-size range would be depleted: if $\mathrm{mm}$-sized grains are present and have grown from the same population of smaller particules, then grains of any smaller sizes should be present as well. Towards the disk midplane, where most of the mass is, only a moderate grain growth, if any, is allowed by the millimeter properties of the disk. Such a grain growth is most likely unsufficient to account for all observations of HK Tau B and would contradict our conclusions regarding the surface of the disk, implicitely making use of a two component (interior/surface) model. Therefore, our analysis points toward an unusual disk structure rather than to unusual dust properties.

A natural way to reconcile the scattered light images with the $1.4 \mathrm{~mm}$ thermal emission map is to assume that both analyses probe different parts of the disk that cannot be extrapolated from one another. So far, we have assumed constant dust properties throughout the disk as well as vertical hydrostatic and isothermal equilibrium. This set of assumptions allowed S98 to infer a total disk mass from the scattered light images which, in fact, only probe the upper and lower surfaces of the disk. If there is a sharp discontinuity in disk properties between those surfaces and the disk interior, where most of the mass lies and most of the thermal emission arises, the validity of this extrapolation breaks down.

We therefore suggest that the disk surrounding HK Tau B has a three-layer structure. The upper and lower surfaces of the disk, above the $\tau_{\lambda}=1$ (as seen from the observer's line of sight) surface at optical/near-infrared wavelength, consist of a light layer of submicron ISM-like dust grains, which are necessary to reproduce the scattered light images. The central layer, which lies around the disk midplane, has a mass in the range $\sim 10^{-3}-2 \times 10^{-2} M_{\odot}$, depending on its temperature (but see Sect. 4.2.3) and the amount of grain growth. These various parameters cannot be distinguished on the sole basis of a single $1.4 \mathrm{~mm}$ flux measurement (e.g., Chiang et al. 2001).

From their fit to the optical image of HK Tau B and to the unresolved $1.3 \mathrm{~mm}$ flux measured by B90, DACH01 concluded to a very different set of disk properties, with grain growth up to $1 \mathrm{~mm}-1 \mathrm{~m}$. Their approach consisted in assuming a disk mass, through an assumed mass accretion rate on the star and disk viscosity properties, and allowing the dust properties to vary until it matched both datasets. Their analysis is weakened by their adopted disk mass which is arguable because HK Tau B displays only moderate signs of accretion for instance. In any case, the fact that this source represents only one third of the $1.4 \mathrm{~mm}$ flux density of the system would readily modify DACH01's conclusions regarding the dust properties. Furthermore, the spectral index of their dust population is $\alpha_{v} \sim 2.9-3.0$, smaller than our derived lower limit. Finally, their fit to the scattered light image was performed with a value of $g$ fixed to the value derived by S98, even though their grain size distribution requires a modified value that would then be 
inconsistent with the data. Overall, we believe that our observations do not agree with their model of the disk mostly because the disk mass and/or structure differs from their assumptions.

Models of three-layer circumstellar disks have already been investigated by several groups (Chiang et al. 2001; DACH01; Malbet et al. 2001) and numerical simulations of the evolution of protoplanetary disks have also resulted in such structures (Suttner \& Yorke 2001). Therefore, our assumption that the disk interior differs from the surface layers is not unphysical. Noticeably, a similar structure was also recently suggested by Weinberger et al. (2002) for the disk surrounding TW Hya on the basis of similar arguments as those presented here. As mentionned above, however, this configuration is not needed to match the observations of several other pre-main sequence disks. This means that the three-layer disk structure discussed above may not be representative of all young circumstellar disks but may rather indicate an important evolutionary process of circumstellar disks. Interestingly, a similar disk structure was suggested for the more massive, though equally young, Herbig AeBe stars by Natta et al. (2001).

\subsection{The disk around HK Tau A}

Our detection of HK Tau A as a millimeter thermal source confirms that it possesses its own circumstellar disk. The fact that we did not resolve it in our $1.4 \mathrm{~mm}$ map suggests that it is smaller than the disk surrounding HK Tau B, although this depends on the surface density and temperature profiles of both disks. In fact, a $100 \mathrm{AU}$ disk with power law indices typical of other T Tauri circumstellar disk $(p+q \gtrsim-2$, D96) would have an apparent size of 70-80 AU at $2.7 \mathrm{~mm}$ (Fig. 4 in D96) and would appear even larger at $1.3 \mathrm{~mm}$ because of the larger dust emissivity and instrument sensitivity. Therefore, the unresolved disk surrounding HK Tau A must be smaller in size than that surrounding HK Tau B, unless it is unusually centrally concentrated. The relatively low spectral index we derived for that source in comparison to other T Tauri circumstellar disks (D96) further suggests that the disk is partially optically thick, which would naturally happen for a small but massive disk, although it may also indicate that large grains are present in this disk.

The smallest disk known around a T Tauri star to date is the 50 AU-radius disk around HV Tau C (Monin \& Bouvier 2000), so this is not an unrealistically small size. However, as the case of HV Tau C illustrates, such a disk would display an obvious central dark lane in scattered light images if it was inclined at $i \sim 84^{\circ}$ as HK Tau B is. To be unresolved in the HST images with such an inclination, the circumstellar disk must be much more compact, with a radius not larger than $\$ 25 \mathrm{AU}$. Still, even though it would not be spatially resolved, such an object would appear much fainter than it actually is since only a small fraction of the stellar flux is scattered towards the observer when a circumstellar disk is seen edge-on. The observed $K$ magnitude of HK Tau A ( $K \approx 8.5$, Kenyon \& Hartmann 1995) places it among the majority of "normal" T Tauri stars in Taurus, while edge-on disk sources in Taurus, such as HK Tau B, are rather characterized by $K \gtrsim 10$ (Stapelfeldt et al. 1997). We therefore conclude that the visible and near-infrared flux from HK Tau A we receive is dominated by direct starlight as opposed to scattered light.

In conclusion, the disk surrounding HK Tau A is not seen edge-on in contrast to the disk observed around HK Tau B. Constraining the inclination of the disk around HK Tau A, however, is not an easy task. Our model shows that the flux from a typical disk around a T Tauri star varies by less than $20 \%$ when its inclination is changed from edge-on to face-on $\left(i=0^{\circ}\right)$. Generally speaking, the observed $1.4 \mathrm{~mm}$ flux density from such a disk is insensitive to its inclination, provided that the latter is $i \lesssim 60^{\circ}$ (see also Wood et al. 2002). This is because the disk is mostly optically thin at this wavelength, so that almost any part of it can be seen from all line-of-sights. Consequently, we can only use the fact that the star is not heavily extincted by its own circumstellar disk and conclude that $i_{\text {prim }} \lesssim 70^{\circ}$, which corresponds to the smallest inclination of currently known flared edge-on disks (Ménard et al. 2002). In other words, the two disks are not parallel to each other and their relative inclination is $\Delta i \gtrsim 15^{\circ}$.

In a number of simulations of the formation of binary systems through fragmentation of an individual molecular core, a driving role is given to rotation or magnetic field. In both cases, the phenomenon is characterized by a prefered/symmetry axis, leading to the formation of two stars with parallel rotation axes, hence parallel disks. In some cases, the disks can be parallel although they do not lie in the orbital plane (Bonnell et al. 1992). This non-coplanar configuration has already been suggested for HK Tau by S98 on the basis of statistical arguments. We are now concluding that the disks are not parallel, which breaks one more symmetry in the system, as the stellar rotation axes are non-parallel either. Capture of two passing-by disked $\mathrm{T}$ Tauri stars is a random process and would naturally result in such a situation, but this is a highly unlikely origin for binary systems in Taurus (e.g., Clarke \& Pringle 1991). On the other hand, recent numerical simulations show that turbulent fragmentation leads to misaligned circumstellar disks for systems wider than $100 \mathrm{AU}$ (M. Bate 2002, priv. comm.), showing that this mechanism can reasonably achieve the configuration observed in HK Tau.

The most puzzling property of the disk surrounding HK Tau A, if confirmed, is the fact that its radius is smaller than that of the less massive secondary component of the system ${ }^{4}$. All theoretical and numerical studies so far have shown that, if the disks' size are driven by the dynamical interaction between the two stellar components and their surrounding material, the more massive star is always surrounded by the largest disk because of its deeper potential well (Artymowicz \& Lubow 1994; Bate \& Bonnell 1997). Our observations therefore suggest that the disk sizes were established during the formation of the binary system rather than through subsequent dynamical evolution.

\footnotetext{
${ }^{4}$ Although pre-main sequence stellar masses are currently strongly model-dependent, the fact that HK Tau B has a somewhat cooler spectral type than HK Tau A (Monin et al. 1998) rules out the possibility that $M_{B}>M_{A}$.
} 


\section{Conclusion}

We have obtained new high angular resolution continuum maps at $1.4 \mathrm{~mm}$ and $2.7 \mathrm{~mm}$ of the pre-main sequence binary system HK Tau. For the first time, the binary is clearly resolved at $1.4 \mathrm{~mm}$ and we have set an upper limit on the flux density of the faintest component at $2.7 \mathrm{~mm}$. The emission from both objects appears to be thermal and, while the $1.4 \mathrm{~mm}$ continuum emission from HK Tau B is resolved along the major axis of its previously known edge-on circumstellar disk, HK Tau A is found to be unresolved at both wavelengths. These observations confirm that both stars are surrounded by their own circumstellar disk. We further find that the disk surrounding HK Tau A must be observed at a less favorable inclination $\left(i \lesssim 70^{\circ}\right)$ than that of its companion, which could be the result of turbulenceinduced fragmentation, while its radius ( $\$ 60 \mathrm{AU})$ is unexpectedly smaller than that of the circumsecondary disk.

Considering the various observational constraints on the circumstellar disk of HK Tau B, we suggest that it must have a layered structure, such as investigated by Chiang et al. (2001). The upper/lower layers, which are displaced vertically from the disk midplane, do not contain a significant population of grains larger than $1 \mu \mathrm{m}$ in radius and thus have dust properties very similar to those of the ISM. On the other hand, the interior of the disk is either massive and constituted of ISM-like grains or has a population of grains that extends significantly beyond $\mu \mathrm{m}$-sized particules. However, the grain size distribution cannot extend to $1 \mathrm{~mm}$ or beyond. Such a disk structure, although suggested for another system, is not required for most disks around T Tauri stars but could naturaly result from dust settling on very short timescales.

Only future high-angular observations of the system covering the mid- and far-infrared ranges will allow a complete study of the disk structure and dust content of HK Tau B, via the analysis of its complete SED. For instance, measurements of the 10-20 $\mu \mathrm{m}$ flux from HK Tau B, where the SED is close to its minimum between the scattering and thermal regimes, would be extremely valuable. NGST and ALMA are among the scheduled new facilities that will allow such observations. A more detailed study of the dust population at the surface of the disk will also be possible through the analysis of visible/nearinfrared polarization maps of the disk.

Acknowledgements. Comments from an anonymous referee helped us significantly improve and clarify this paper. Part of this work was supported by the Programme National de Physique Stellaire of the Institut National des Sciences de l'Univers. This research has made use of the SIMBAD database, operated at CDS, Strasbourg, France.

\section{References}

Artymowicz, P., \& Lubow, S. 1994, ApJ, 421, 651

Bate, M., \& Bonnell, I. 1997, MNRAS, 285, 33

Beckwith, S., Sargent, A., Chini, R., \& Guesten, R. 1990, AJ, 99, 924 (B90)

Beckwith, S., \& Sargent, A. 1991, ApJ, 381, 250 (BS91)

Bertout, C., Robichon, N., \& Arenou, F. 1999, A\&A, 352, 574

Bonnell, I., Arcoragi, J.-P., Martel, H., \& Bastien, P. 1992, ApJ, 400, 579

Burrows, C., Stapelfeldt, K., Watson, A., et al. 1996, ApJ, 473, 437

Chiang, E., Joung, M., Creech-Eakman, M., et al. 2001, ApJ, 547, 1077

Clarke, C., \& Pringle, J. 1991, MNRAS, 249, 584

Cohen, M., \& Kuhi, L. 1979, ApJS, 42, 743

D'Alessio, P, Calvet, N., \& Hartmann, L. 2001, ApJ, 553, 321 (DACH01)

Duchêne, G. 1999, A\&A, 341, 547

Dutrey, A., Guilloteau, S., Duvert, G., et al. 1996, A\&A, 309, 493 (D96)

Guilloteau, S., Delannoy, J., Downes, D., et al. 1992, A\&A, 262, 624

Guilloteau, S., Dutrey, A., \& Simon, M. 1999, A\&A, 348, 570

Henning, T., \& Stognienko, R. 1996, A\&A, 311, 291

Herbig, G., \& Bell, R. 1988, Lick Obs. Bull., 1111

Jensen, E., Donnar, A., \& Mathieu, R. 2000, in Birth and evolution of binary stars, ed. B. Reipurth, \& H. Zinnecker, Poster Proc. of IAU Symp., 200, 85

Kenyon, S., \& Hartmann, L. 1995, ApJS, 101, 117

Kim, S.-H., Martin, P., \& Hendry, P. 1994, ApJ, 422, 164

Koresko, C. 1998, ApJ, 507, L145 (K98)

Krist, J., Stapelfeldt, K., \& Watson, A. 2002, ApJ, 570, 785

Lagrange, A.-M., Backman, D., \& Artymowicz, P. 2000, in Protostars and planets IV, ed. V. Mannings, A. P. Boss, \& S. S. Russell (Univ. of Arizona Press), 39

McCabe, C., Duchêne, G., \& Ghez, A. 2002, ApJ, 575, 974

Malbet, F., Lachaume, R., \& Monin, J.-L. 2001, A\&A, 379, 515

Mathis, J., \& Whiffen, G. 1989, ApJ, 341, 808

Ménard, F., Dougados, C., Magnier, E., et al. 2002, ApJ, submitted

Miyake, K., \& Nakagawa, Y. 1993, Icarus, 106, 20

Moneti, A., \& Zinnecker, H. 1991, A\&A, 242, 428

Monin, J.-L., Ménard, F., \& Duchêne, G., A\&A, 339, 113

Monin, J.-L., \& Bouvier, J. 2000, A\&A, 356, L75

Natta, A., Prusti, T., Neri, R., Wooden, D., et al. 2001, A\&A, 371, 186

O’Neal, D., Feigelson, E., Mathieu, R., \& Myers, P. 1990, AJ, 100, 1610

Pollack, J., Hollenbach, D., Beckwith, S., et al. 1994, ApJ, 421, 615

Stapelfeldt, K., Burrows, C., Krist, J., \& the WFPC2 Science Team 1997, in Herbig-Haro flows and the birth of stars, ed. B. Reipurth, \& C. Bertout (Kluwer), IAU Symp., 182, 355

Stapelfeldt, K., Krist, J., Ménard, F., et al. 1998, ApJ, 502, L65 (S98)

Suttner, G., \& Yorke, H. 2001, ApJ, 551, 461

Weinberger, A., Becklin, E., Schneider, G., et al. 2002, ApJ, 566, 409

Witt, A., Oliveri, M., \& Schild, R. 1990, AJ, 99, 888

Wood, K., Wolff, M., Bjorkman, J., \& Whitney, B. 2002, ApJ, 564, 887

Zubko, V., Krelowski, J., \& Wegner, W. 1998, MNRAS, 294, 548 